\title{
Predicting the Potential Distribution of Endangered and Rare Satyrium Species in China under Climate Change
}

Xianheng Ouyang ${ }^{1}$, Shihao Bai ${ }^{2}$, Anliang Chen ${ }^{1}$, Garry Brien Strachan ${ }^{1}$, Luying Zuo ${ }^{1}$, and Yang Yang ${ }^{3}$

${ }^{1}$ Zhejiang $\mathrm{A}$ and $\mathrm{F}$ University

${ }^{2}$ Shanghai Jiao Tong University

${ }^{3}$ Hunan University of Medicine

November 5, 2021

\begin{abstract}
Satyrium is an endangered and rare genus of plant that has various pharmacodynamic functions. The prediction of potentially suitable habitat of Satyrium species and to analyze their changes, the model MaxEnt niche was applied; furthermore, the receiver operating characteristic curve was applied to analyze accuracy. The results provided an available reference for implementation of long-term conservation and management approaches for the species. Under the current climatic conditions, the area of the total suitable habitat for Satyrium ciliatum (S. ciliatum) in China is $32.51 \times 104 \mathrm{~km} 2$, the total suitable habitat area for Satyrium nepalense (S. nepalense) in China is $61.76 \times 104 \mathrm{~km} 2$, and the area of the total suitable habitat for Satyrium yunnanense (S. yunnanense) in China is $89.73 \times 104 \mathrm{~km} 2$. The potential suitable habitat of Satyrium is mainly distributed in Southwest China. The major environmental changes influencing the geographical distribution of S. ciliatum were temperature seasonality (Bio4), isothermality (Bio3), and mean temperature of coldest quarter (Bio11). The geographical distribution of S. nepalense was mainly affected by environmental variables like temperature seasonality (Bio4), isothermal environment (Bio3), and precipitation of the coldest quarter (Bio19); and the geographical distribution of the species S. yunnanense were mainly affected by the environmental variables such as temperature seasonality (Bio4), isothermality (Bio3) and lower temperature of the coldest month (Bio6). The distribution range of Satyrium's extended with global warming increased, in both situations' emissions of greenhouse gases with lower concentration (SSP1-2.6) and the emission of greenhouse gases with higher concentration (SSP5-8.5). According to the study, the distribution of suitable habitat would shift with change to higher elevated areas in the coming time.
\end{abstract}

Predicting the Potential Distribution of Endangered and RareSatyrium Species in China under Climate Change

Xianheng Ouyang ${ }^{1}$ and Shihao Bai $^{2}$, Anliang Chen ${ }^{1 *}$, Garry Brien Strachan ${ }^{3}$,Luying Zuo ${ }^{1}$, Yang Yang ${ }^{4}$

1 School of Forestry and Biotechnology, Zhejiang A\&F University, Hangzhou 311300, China

2 Shanghai Center for Systems Biomedicine, Shanghai Jiao tong University, Shanghai 200240, China

3 School of Law and Humanities, Zhejiang A\&F University, Hangzhou 310018, China

4 School of Pharmaceutical Sciences, Hunan University of Medicine, Huaihua, 418000, China

Correspondence

Anliang Chen, School of Forestry and Biotechnology, Zhejiang A\&F University, Hangzhou 311300, China. 


\section{Email: anlchen@126.com}

\section{Acknowledgements}

The work was supported by Science and Technology Plan Projects of Zhejiang Province [grant numbers 2019C02024].

Abstract: Satyrium is an endangered and rare genus of plant that has various pharmacodynamic functions. The prediction of potentially suitable habitat of Satyrium species and to analyze their changes, the model MaxEnt niche was applied; furthermore, the receiver operating characteristic curve was applied to analyze accuracy. The results provided an available reference for implementation of long-term conservation and management approaches for the species. Under the current climatic conditions, the area of the total suitable habitat forSatyrium ciliatum (S. ciliatum) in China is $32.51 \times 10^{4} \mathrm{~km}^{2}$, the total suitable habitat area for Satyrium nepalense ( $S$. nepalense) in China is $61.76 \times 10^{4} \mathrm{~km}^{2}$, and the area of the total suitable habitat for Satyrium yunnanense ( $S$. yunnanense) in China is $89.73 \times 10^{4} \mathrm{~km}^{2}$. The potential suitable habitat of Satyrium is mainly distributed in Southwest China. The major environmental changes influencing the geographical distribution of S. ciliatum were temperature seasonality (Bio4), isothermality (Bio3), and mean temperature of coldest quarter (Bio11). The geographical distribution of $S$. nepalense was mainly affected by environmental variables like temperature seasonality (Bio4), isothermal environment (Bio3), and precipitation of the coldest quarter (Bio19); and the geographical distribution of the species S. yunnanense were mainly affected by the environmental variables such as temperature seasonality (Bio4), isothermality (Bio3) and lower temperature of the coldest month (Bio6). The distribution range of Satyrium's extended with global warming increased, in both situations' emissions of greenhouse gases with lower concentration (SSP1-2.6) and the emission of greenhouse gases with higher concentration (SSP5-8.5). According to the study, the distribution of suitable habitat would shift with change to higher elevated areas in the coming time.

Keywords: Satyrium, MaxEnt niche, the potential suitable habitat, environmental variables.

Global warming is one of the crucial environmental problems facing the world today (1). The global temperature has risen by about 1 in the past century, especially in the past 30 years, causing some florae species to move to higher altitude areas, as reported by the Fifth Assessment Report (AR5) of the United Nations Intergovernmental Panel on Climate Change (2). According to the different suggestions that the future change in climate has an impact on the distribution range of species, resulting in the loss of biodiversity and the endangered species will be extinct (3). After climate change, the prediction of the appropriate habitat of species was considered important in the future (4). In research on the geographical distribution range of plants affected by climate change, the species distribution model (SDM) uses the distribution data of species and environmental variables in their habitat to predict the basic niche of species. These data are combined with environmental data in different periods for simulating the potential distribution areas of species in those periods (5). Among many SDMs, the maximum entropy model (MaxEnt) has the advantages of fast modeling, wide use and high accuracy, and stability even with a small sample size $(6,7)$. It can better anticipate the possible distribution of species compared with other SDMs (8).

Satyrium (Orchidaceae) is rare and endangered plant genus with about 92 species, mostly found on continents of Africa, 5 species found in Madagascar and 4 species are found in Asian countries (9). Just 3 species are found in China alone, namely, Satyrium ciliatum(S. ciliatum ), Satyrium nepalense (S. nepalense), and Satyrium yunnanense (S. yunnanense ) (10).Satyrium is also used for traditional herbal medicinal purposes; such as the traditional healthcare centers in India used the tubers of $S$. nepalense to make energy tonics and it is mostly used for curing different types of fevers.

The number of Satyrium resources has been rapidly diminishing. Its population has a fragmented distribution and is in a rare and endangered state. It is facing extinction because of the value of herbal medicine and the serious deterioration of the ecological environment in recent times (9). The changes in climate will result in changes in the biological phenology period. This in turn will result in changes in the geographical distribution of species and a quickening of the rate of species extinction. Therefore, it is necessary to 
adequately understand the changing trends of the geographical distribution of species under the conditions of climate change and to develop relative protection strategies.

The diversity of Satyrium species were predicted by employing the MaxEnt model:

(1) In current climatic conditions, the geographical diversity ofSatyrium species in China were studied, and relationship between these distributions and environmental changes were studied. The main environmental variables limiting their potential geographically diversity was outlined.

(2) The potential geographical distribution and shifting trend of the centroid of suitable areas in China were predicted under the scenario of future climate change. To offer a scientific foundation for the appropriate protection and utilization of Satyrium, the conceivable geographical distribution, dynamic change features, and affecting environmental elements in China under different climate conditions were defined.

\section{Materials and Methods}

\subsection{Data of the Species occurrence}

The distribution data of $S$. ciliatum , S. nepalense, andS. yunnanense in China were obtained from the National Specimen Information Infrastructure (http:// www. nsii. org. cn/), the National Plant Specimen Resource Center (http://www.cvh.ac.cn/) and the Global Biodiversity Information Fcility (https:www.gbif.org/). The geographical coordinates were not recorded in detail in some distribution points. Thus, Google Earth (http://ditu.google.cn/) was used to obtain the geographical coordinates of the points. Points with repeated latitude and longitude and missing points with latitude and longitude were deleted. In the study, a total of 44, 20, and 16 distribution data samples of S. ciliatum, S. nepalense, and S. yunnanense were gathered and utilized to run the MaxEnt model simulation (Fig. 1).

\subsection{Environmental variables}

A total of 22 variables were studied including 19 climatic variables, 3 topographical variables, and 22 initial environmental variables with a resolution of 2.5' (Table S1). The three topographic parameters (alt, slope, and asp) were downloaded with a resolution of 2.5' from World Climate data (http://www.worldclim.org/). Bioclimatic characteristics obtained from the WorldClim (version 2.0) database were utilized to build distribution prediction models (11). The predictions for the Geographical distributions of S. ciliatum, $S$. nepalense, and $S$. yunnanense were carried out by the BCC-CSM2-MR climate system model, developed by the National Climate Center (12). The World Climate Database (http://www.worldclim.org/ ) was used for downloading the future climate data 2050 (2041-2060) and 2070 (2061-2080). New future pathways based on socioeconomic assumptions are called Shared Socioeconomic Pathways (SSPs) that describe various levels of socioeconomic development. These were used in the study (13). The SSPs include the high-forcing scenario (SSP5-8.5), the medium-forcing scenario (SSP2-4.5), and the low-forcing scenario (SSP1-2.6). The high and low emission scenarios are represented by the SSP5-8.5 and SSP1-2.6 in the study, respectively.

It is necessary to analyze environmental variables before they can be used for niche simulation calculations to avoid the multicollinearity of the variables causing over-fitting of the model because many bioclimatic variables are spatially related (14). ArcGIS10.2 was employed to examine the relationships between these 22 bioclimatic variables (Table S1). When the correlation coefficient between two climatic variables was more than 0.80 , climate variables of greater ecological relevance were kept. For the prediction of the distribution of $S$. ciliatum, S. nepalense, and S. yunnanense, a total of 7,9 , and 8 variables with coefficients greater than 0.8 (15) were maintained, respectively (Fig. 2).

\subsection{MaxEnt model ensembles}

MaxEnt software (http://www.cs.princeton.edu/, version 3.4.1) was employed for creating the Satyrium habitat suitability model. Models that produced $75 \%$ of the occurrence records were used for training, while those that produced $25 \%$ of the occurrence records were employed for testing (16).

The number of iterations and the number of background points were both limited to 1000 and 10,000, 
respectively. The model's performance was improved by repeating the ensemble processes using 10 -fold crossvalidation (8). The logistical output of the MaxEnt software, which revealed environmental appropriateness for S. ciliatum, S. nepalense, and S. yunnanense with values no higher than one, was used to create the final prospective distribution map. For additional analysis, the MaxEnt results were combined in ArcGIS 10.2, and the prospective habitat categories were regrouped into four groups: highly suitable habitat (0.50-1.00), moderately habitat (0.30-0.50), poorly suitable habitat (0.10-0.30), and unsuitable habitat (0-0.10).

\subsection{Evaluation of the MaxEnt Model}

Data from the receiver operating characteristic (ROC) curve (17) were applied to aids the model's accuracy. It is shown that model simulation results were excellent when the values of the area under the AUC (area under the receiver operating characteristics) (ROC) curve were $>0.9(8,18)$.

\subsection{Core distributional shifts}

Under current and future climate circumstances, the SDM toolbox is used to calculate shifting trends in the area of appropriate habitat based on Python, as well as changes in the centroid of the area of suitable habitat (19). The toolbox is a Python-based GIS application (20). Using the toolbox, researchers calculated shifting trends in areas of appropriate habitats and compared the centroids of the regions between current and future climate conditions. The study provides information about the core shifts and distribution of S. ciliatum, $S$. nepalense, and S. yunnanense. These species' diversity was reduced to a single centroid (central) point, and a vector file was constructed to show the amount and direction of expected change over time. Finally, we looked at how the centroid changed with different SDMs to see if there were any distribution shifts.

\section{Results}

\subsection{SDM and its accuracy}

The ROC curve was used to assess the potential distributions of $S$. ciliatum, S. nepalense, and S. yunnanense , and the AUC values of training data were $0.988,0.981$, and 0.958 , respectively (Fig. 3). This showed that the distribution records of $S$. ciliatum, S. nepalense, and S. yunnanense predicted based on the MaxEnt model were not random, and the prediction of the model was reliable.

\subsection{Important environmental variables}

The choice of environmental variables is the key to determine the accuracy of modeling. Therefore, it is necessary to evaluate which environmental variables contribute the most to the model. Temperature was the main environmental variable affecting S. ciliatum, S. nepalense, and $S$. yunnanense, according to the MaxEnt model's variable contribution rate (Fig. 2). Bio3(52\%) and Bio4(12.7) for S. ciliatum reached $64.7 \%$. Furthermore, Bio3 (61.8\%) and Bio4 (14\%), both for S. nepalense, achieved $75.6 \%$, much greater than the other contribution rates. Bio3(28.6\%) and Bio6(54.3\%) for S. yunnanense were $72.9 \%$. This reveals that Bio3, Bio4, and Bio6 were the most important influencing variables in S. ciliatum, S. nepalense, and $S$. yunnanense distribution.

The Jackknife test was used to determine the impact of environmental variables on S. ciliatum, S. nepalense, and $S$. yunnanensepotential distribution (Fig. 4). When a single variable was applied in relation to $S$. ciliatum, the jackknife test showed that the most influential environmental variable is Bio4 followed by Bio3 and Bio11. When a single variable was applied in relation to $S$. nepalense, the most influential variable was Bio3 followed by Bio4 and Bio19. When a single environmental variable was applied in relation to $S$. yunnanense, the most influential variable was Bio4 followed by Bio6 and Bio3. The most important variables influencing S. ciliatumdistribution were Bio3, Bio4, and Bio11. S. nepalensedistribution was influenced by the variables were Bio4, Bio3, and Bio19. Bio4, Bio3, and Bio6 are the most important variables influencing the distribution of $S$. yunnanense.

The response curve was used to calculate the thresholds (existence probability $>0.5$ ) for the main bioclimatic parameters (Fig. 5). In relation to S. ciliatum, Bio3 ranged from 44 to 51.5, Bio4 ranged from 420 to 580, and Bio11 ranged from 1 to 13. 
In relation to S. nepalense. Bio3 ranged from 44 to 53, Bio4 ranged from 400 to 580, and Bio19 ranged from 25 to $80 \mathrm{~mm}$.

With regard to S. yunnanense, Bio3 ranged from 41 to 53, Bio4 ranged from 300 to 600, and Bio6 ranged from -6 to 6 .

\subsection{Potential distribution of Satyrium ciliatum, Satyrium nepalense, and Satyrium yunna- nense.}

S. ciliatum are mainly distributed in Southwest China (Fig. 6). Highly suitable habitats are located at the border of Yunnan and Sichuan. Eastern Yunnan and Southern Sichuan have moderately appropriate environments. Poorly appropriate habitats cover a broad area, primarily in Northern and Central Yunnan, Southern Tibet, Western Sichuan, and Western Guizhou. There is also a sporadic distribution in Western Guangxi.

S. nepalense are mainly distributed in Southwest China. Highly suitable habitats are distributed in Central Yunnan. Western and eastern Yunnan, as well as southern Sichuan, have moderately appropriate environments. Poorly suitable habitats are located in Southern Yunnan, Western and Southern Sichuan, Southern Tibet, Western Sichuan, and Western Guangxi.

S. yunnanense are mainly distributed in Southwest China. Highly suitable habitats are distributed in Yunnan. Moderately suitable habitats are located at the border of Yunnan, Guizhou, and Sichuan. Poorly suitable habitats are distributed in Western Guizhou, Central Sichuan, Southern Shaanxi, Western Henan, Southern Fujian, Southern Tibet, and Western Guangxi.

The total suitable area for $S$. yannanense is more than that of $S$. nepalense and $S$. ciliatum according to the mentioned climatic conditions (Table 1). These areas account for $9.35 \%, 6.43 \%$ and $3.39 \%$, respectively, the total land area of china $\left(960 \times 10^{4} \mathrm{~km}^{2}\right)$ (Table 1$)$. The highly suitable habitat area for S. ciliatum in the 2050s for SSP1-2.6, will be $9.92 \times 10^{4} \mathrm{~km}^{2}$, the moderately suitable habitat area will be $11.39 \times 10^{4} \mathrm{~km}^{2}$, and the total habitat area will be $49.36 \times 10^{4} \mathrm{~km}^{2}$. In the 2050s, for SSP5-8.5, the hilghly suitable habitat area for S. ciliatum's will be $12.19 \times 10^{4} \mathrm{~km}^{2}$, the moderately suitable habitat will be $12.02 \times 10^{4} \mathrm{~km}^{2}$, and the total area for habitat will be $51.83 \times 10^{4} \mathrm{~km}^{2}$. In the 2070s,for SSP1-2.6, the $9.74 \mathrm{x} 10^{4} \mathrm{~km}^{2}$ area will be a highly suitable habitat for $S$. ciliatum species, the moderately suitable habitat will be $11.36 \times 10^{4} \mathrm{~km}^{2}$, and $48.1 \times 10^{4} \mathrm{~km}^{2}$ will be the total habitat area. In the 2070s, for SSP5-8.5, the highly suitable habitat area for S. ciliatum's will be $21.94 \mathrm{x} 10^{4} \mathrm{~km}^{2}$, the moderately suitable habitat is will be $15.34 \mathrm{x} 10^{4} \mathrm{~km}^{2}, 69.46$ $\mathrm{x} 10^{4} \mathrm{~km}^{2}$ will be the total area for habitat. The habitat which is more suitable will be found in Sichuan, Yunnan, Southern Tibet, and the border between Chongqing and Sichuan (Fig. 07).

The area of the highly appropriate habitat of $S$. nepalense in the 2050 s for SSP1-2.6 will be $13.07 \times 10^{4} \mathrm{~km}^{2}$, the area of moderately suitable habitat will be $18.53 \times 10^{4} \mathrm{~km}^{2}$, and the area of total habitat will be 76.42 x $10^{4} \mathrm{~km}^{2}$. In the 2050s, for SSP5-8.5, the area of $S$. nepalense highly appropriate habitat will be $11.95 \mathrm{x}$ $10^{4} \mathrm{~km}^{2}$, the area of moderately suitable habitat will be $18.29 \times 10^{4} \mathrm{~km}^{2}$, and the area of total habitat will be $74.75 \times 10^{4} \mathrm{~km}^{2}$. The amount of highly appropriate habitat for S. nepalense in the 2070s for SSP1-2.6 will be $11.51 \times 10^{4} \mathrm{~km}^{2}$, the area of moderately suitable habitat will be $18.98 \times 10^{4} \mathrm{~km}^{2}$, and the area of total habitat will be $75.15 \times 10^{4} \mathrm{~km}^{2}$. In the 2070s, for SSP5-8.5, the area of S. nepalense highly suitable habitat will be $16.60 \times 10^{4} \mathrm{~km}^{2}$, the area of moderately suitable habitat will be $27.43 \times 10^{4} \mathrm{~km}^{2}$, and the area of total habitat will be $97.52 \times 10^{4} \mathrm{~km}^{2}$. It will mostly be located in Yunnan, Sichuan, Southern Tibet, and along the border between Sichuan and Chongqing (Fig. 7).

In the 2050s, for SSP1-2.6, the total highly suitable area for the habitat of S. yunnanense will be $20.91 \mathrm{x}$ $10^{4} \mathrm{~km}^{2}, 21.84 \times 10^{4} \mathrm{~km}^{2}$ area will be moderately suitable habitat, and the total habitat area will be 123.31 x $10^{4} \mathrm{~km}^{2}$. In the 2050s, for SSP5-8.5, $18.25 \times 10^{4} \mathrm{~km}^{2}$ area will be highly suitable for the habitat of $S$. yunnanense, the moderately suitable habitat area will be $24.31 \mathrm{x} 10^{4} \mathrm{~km}^{2}$, and the total habitat area will be $124.33 \times 10^{4} \mathrm{~km}^{2}$ (Fig.07). In the 2070s, for SSP1-2.6, the area of highly appropriate habitat for $S$. yunnanense will be $19.58 \times 10^{4} \mathrm{~km}^{2}$, the amount of moderately suitable habitat will be $23.60 \times 10^{4} \mathrm{~km}^{2}$, and 
the total habitat area will be $111.53 \times 10^{4} \mathrm{~km}^{2}$. In the 2070s, for SSP5-8.5, the area of $S$. yunnanense highly appropriate habitat will be $25.12 \times 10^{4} \mathrm{~km}^{2}$, the area of moderately suitable habitat will be $31.79 \times 10^{4} \mathrm{~km}^{2}$, and the area of total habitat will be $163.3 \times 10^{4} \mathrm{~km}^{2}$. The majority of the region will be in Yunnan, the middle of Sichuan, Southern Tibet, and the border between Sichuan and Chongqing (Fig. 7).

\subsection{Future changes in appropriate habitat areas and variations in highly suitable centroid distribution.}

According to Table 2, using SSP1-2.6, the lost area for S. ciliatum in the 2050 s will be $0.06 \times 10^{4} \mathrm{~km}^{2}$, the acquired area will be $9.65 \times 10^{4} \mathrm{~km}^{2}$, and the stable area will be $10.79 \times 10^{4} \mathrm{~km}^{2}$. The gained territory will be in Southern Tibet and Northwest Sichuan. According to SSP5-8.5, the lost area for S. ciliatum in the 2050 s will be $0.09 \times 10^{4} \mathrm{~km}^{2}$, the acquired area will be $12.63 \times 10^{4} \mathrm{~km}^{2}$, and the stable area will be $10.74 \mathrm{x}$ $10^{4} \mathrm{~km}^{2}$. The gained territory will be in Southern Tibet and Northwest Sichuan. The new territory will be in Southern Tibet, Northwest Sichuan, and Northern Yunnan. Under SSP1-2.6, the lost area for S. ciliatum in the 2070s will be $0.07 \times 10^{4} \mathrm{~km}^{2}$, the gained area will be $9.56 \times 10^{4} \mathrm{~km}^{2}$, and the stable area will be $10.75 \times 10^{4} \mathrm{~km}^{2}$. The new territories will be in Southern Tibet, Central Yunnan, and Northwest Sichuan. According to SSP5-8.5, the lost area for S. ciliatum in the 2070 s will be $0.21 \times 10^{4} \mathrm{~km}^{2}$, the acquired area will be $26.33 \times 10^{4} \mathrm{~km}^{2}$, and the stable area will be $10.48 \times 10^{4} \mathrm{~km}^{2}$. The new territory will be in Southern Tibet, Northeast Yunnan, and Northwest Sichuan (Fig. 8).

Using SSP1-2.6, the lost area for S. nepalense in the 2050s will be $0.36 \times 10^{4} \mathrm{~km}^{2}$, the gained area will be $13.43 \times 10^{4} \mathrm{~km}^{2}$, and the stable area will be $62.53 \times 10^{4} \mathrm{~km}^{2}$. The gained territory will be in Southern Tibet and Northwest Sichuan. According to SSP5-8.5, the lost area for S. nepalense in the 2050s will be $0.77 \times 10^{4} \mathrm{~km}^{2}$, the acquired area will be $12.42 \times 10^{4} \mathrm{~km}^{2}$, and the stable area will be $61.97 \times 10^{4} \mathrm{~km}^{2}$. The gained territory will be in Southern Tibet and Northwest Sichuan. According to SSP1-2.6, the lost area for S. nepalense in the $2070 \mathrm{~s}$ will be $0.60 \times 10^{4} \mathrm{~km}^{2}$, the gained area will be $12.70 \times 10^{4} \mathrm{~km}^{2}$, and the stable area will be $62.13 \times 10^{4} \mathrm{~km}^{2}$. The gained territory will be in Southern Tibet and Northwest Sichuan. Under SSP5-8.5, the lost area for S. nepalense in Southern Tibet, Northwest Sichuan, and Southwest Gansu will be $1.63 \times 10^{4} \mathrm{~km}^{2}$, the gained area will be $36.86 \times 10^{4} \mathrm{~km}^{2}$, and the stable area will be $60.77 \times 10^{4} \mathrm{~km}^{2}$ in the 2070s (Fig. 8).

According to SSP1-2.6, the lost area for S. yunnanense in the 2050s will be $2.38 \times 10^{4} \mathrm{~km}^{2}$, the gained area will be $34.15 \times 10^{4} \mathrm{~km}^{2}$, and the stable area will be $88.65 \times 10^{4} \mathrm{~km}^{2}$. Southern Shaanxi, Southern Shanxi, Northern Henan, and Central Shandong will be the new provinces. According to SSP5-8.5, the lost area for S. yunnanense in the $2050 \mathrm{~s}$ will be $7.50 \times 10^{4} \mathrm{~km}^{2}$, the acquired area will be $41.16 \times 10^{4} \mathrm{~km}^{2}$, and the stable area will be $83.05 \times 10^{4} \mathrm{~km}^{2}$. Southwestern Xinjiang, Southern Tibet, Northern Sichuan, Southern Shaanxi, Southern Gansu, Northern Henan, and Central Shandong will gain territory. According to SSP1-2.6, the lost area for $S$. yunnanense in the 2070 s will be $6.18 \times 10^{4} \mathrm{~km}^{2}$, the gained area will be $26.87 \times 10^{4} \mathrm{~km}^{2}$, and the stable area will be $84.47 \times 10^{4} \mathrm{~km}^{2}$. Southern Xinjiang, Southern Tibet, Northern Sichuan, Northern Henan, and Southern Shandong will gain territory. According to SSP5-8.5, the lost area for S. yunnanense in the 2070s will be $15.69 \times 10^{4} \mathrm{~km}^{2}$, the acquired area will be $89.29 \times 10^{4} \mathrm{~km}^{2}$, and the stable area will be $74.35 \times 10^{4} \mathrm{~km}^{2}$. Southern Xinjiang, Southern Tibet, Northern and Eastern Guizhou, Southern Gansu, Southern Ningxia, Southern Shaanxi, Southern Hebei, and Western Shandong would gain territory (Fig. 8).

\subsection{Core distributional shifts}

Fig. 9 shows that the centroid of S. ciliatum's current habitat is located in Central Yunnan Province at $99 \mathrm{deg} 84^{\prime} \mathrm{E}$ and $27 \mathrm{deg} 27^{\prime} \mathrm{N}$. Under SSP1-2.6, the centroid of appropriate habitat will shift to 99deg35'E and $27 \mathrm{deg} 87^{\prime} \mathrm{N}$ in the $2050 \mathrm{~s}$, and $98 \mathrm{deg} 69^{\prime} \mathrm{E}$ and $27 \mathrm{deg} 94{ }^{\prime} \mathrm{N}$ in the $2070 \mathrm{~s}$. Under SSP5-8.5, the centroid of the future appropriate region is located at $98 \mathrm{deg} 86{ }^{\prime} \mathrm{E}$ and $28 \mathrm{deg} 01^{\prime} \mathrm{N}$ in the $2050 \mathrm{~s}$. Under SSP $5-8.5$, the acceptable area's centroid will relocate to $98 \mathrm{deg} 15$ 'E and $28 \mathrm{deg} 91$ ' $\mathrm{N}$ in the $2070 \mathrm{~s}$. In summary, we can see that the core distribution of $S$. ciliatum will shift to the northwest under both future emission trajectories (SSP1-2.6, SSP5-8.5).

The centroid of the existing habitat for S. nepalense is located in Southern Yunnan Province at 99deg28'E 
and $27 \mathrm{deg} 15$ 'N. (Fig. 9). Under SSP1-2.6, the centroid of the appropriate region will shift to 98deg79'E and $27 \operatorname{deg} 69^{\prime} \mathrm{N}$ in the $2050 \mathrm{~s}$, and $98 \mathrm{deg} 38^{\prime} \mathrm{E}$ and $27 \operatorname{deg} 69^{\prime} \mathrm{N}$ in the $2070 \mathrm{~s}$. Under SSP5-8.5, the centroid of the future appropriate region is located at $98 \mathrm{deg} 82^{\prime} \mathrm{E}$ and $27 \mathrm{deg} 69^{\prime} \mathrm{N}$ in the $2050 \mathrm{~s}$. The centroid of the appropriate region will relocate to $97 \operatorname{deg} 67^{\prime} \mathrm{E}$ and $28 \mathrm{deg} 56^{\prime} \mathrm{N}$ in the $2070 \mathrm{~s}$ under SSP 5-8.5.

The centroid of the current habitat for S. yunnanense is located in South Sichuan Province at 102deg67'E and $27 \mathrm{deg} 75^{\prime} \mathrm{N}$. (Fig. 9). Under SSP1-2.6, the centroid of the appropriate area will shift to $103 \mathrm{deg} 73^{\prime} \mathrm{E}$ and $29 \operatorname{deg} 02^{\prime} \mathrm{N}$ in the $2050 \mathrm{~s}$, and $102 \mathrm{deg} 22^{\prime} \mathrm{E}$ and $28 \operatorname{deg} 94^{\prime} \mathrm{N}$ in the $2070 \mathrm{~s}$. According to SSP5-8.5, the acceptable area's centroid will relocate to $101 \operatorname{deg} 91^{\prime} \mathrm{E}$ and $29 \operatorname{deg} 68^{\prime} \mathrm{N}$ by $2050 \mathrm{~s}$, then to $100 \mathrm{deg} 05^{\prime} \mathrm{E}$ and 30 $\operatorname{deg} 94$ ' $\mathrm{N}$ in the $2070 \mathrm{~s}$.

\section{Discussion}

In plant species the Satyrium species are endangered and rare which is the important resources (21). MaxEnt model were applied to records the distribution and predict the potential distribution areas of $S$. ciliatum, S. nepalense, and $S$. yannanense. The values for training data by AUC were $0.988,0.981$ and 0.958 , and it shows that MaxEnt predictions is valid. According to the four different future scenarios, that the suitable habitat area for Satyrium'sin China will extend in 2050s and 2070s, and the suitable habitat area will move slowly to the higher altitude area in Northwest side of China, where the humidity and habitat is suitable for the growth ofSatyrium species. The prediction shows the in upcoming future will be the most suitable for the growth for Satyrium species. Its shows that generally the suitable habitat is stable and cause of the climatic change the species will face no extinctions. The study will show the new areas for the species distribution. The findings were in good agreement with the findings of the studies published peviosuly, thus indicating that, in some regions, habitat suitability of plant species improves under climate change conditions $(22,23)$. The area of species covering $20 \%$ of the earth's surface will face the risk of extinction (24). About $15 \%-37 \%$ of the species will be endangered, as predicted by the distribution of the species under the future climate scenario. One of the reasons for the endangered status of Satyrium is that most Satyrium species are hermaphroditic. Some species rely on birds and other animals for long-distance transmission. The species population is small, and the quality of seed development is low because of long-distance pollination (25). This study's results provide a reliable basis for the protection of Satyrium. People should effectively protect its native habitat and population. Nature reserves of Satyrium should be set up now according to the prediction results, and they should be strengthened to minimize human-made damage.

The results of the current study, using the response curve, obtained the thresholds (existence probability $>$ 0.5 ) for the main bioclimatic parameters. Regarding S. ciliatum, isothermality (Bio3) ranged from 44 to 51.5 , temperature seasonality (Bio4) ranged from 420 to 580, and mean temperature of the coldest quarter (Bio11) ranged from 1 to 13 . Temperature seasonality (Bio4) ranged from 400-580, Isothemality (Bio3) ranged from 44-53 and precipitation of the coldest quarter (Bio19) ranged from 25-80mm for S.nepalense. Temperature seasonality (Bio4) ranged from $300-600 \mathrm{~mm}$, Isothermility (Bio3) ranged from 41-53 and lower temperature of coldest month(Bio06) from -6-6degC forS. yunnanense. This shows that the main environmental factors affecting the suitable distribution of Satyrium are temperature and precipitation. The plant distribution patterns can be detected by the environmental factors like temperature and precipitation (26, 27). Geographical distribution of species can be determined by the climate change and geographical distribution of species also response to change in climate (28). The structures of terrestrial ecosystems have been changed by global warming, and the habitat and geographical distribution functions of species have been changed by the structure of terrestrial ecosystems in turn (29). For instance, climate, topography, soil, human disturbance and spatial constraints are significant to the distribution of many different spatial scales(30). More studies have shown that the dominant variables restricting geographical distribution of plants are energy supply needed to complete life cycles, plenty of water, and cold tolerance (31). Based on the prediction of the MaxEnt model, the area of the current potential suitable habitat of Satyrium mainly located in Southwest China, which has a typical subtropical monsoon climate (32). Previous studies have indicated that precipitation is the main varaible affecting plants growth, regeneration, nutrient cycles, and community productivity in different habitats $(33,34)$. The environmental data used in the study just in- 
cluded the bioclimatic variables in WorldClim. We did not study soil type, land use, human activities, biological interaction, and other factors that influence the distribution of Satyrium . Certainly, the more factors that are included, the more accurate the prediction will be. Therefore, other factors affecting the potential suitable habitat of Satyriumshould be studied in the future, with different niche prediction models predicting the areas of the potential suitable habitats. This will make the prediction of results more reliable.

\section{Conclusion}

Precipitation (Bio19) and temperature (Bio4, Bio3, Bio6 \& Bio11) are the main environmental factors for the potential geographical distribution of Satyrium's. The potential geographical distribution of Satyrium's under recent climatic conditions are primarily in southwest sides of China. Satyrium's highly suitable habitat area, moderately suitable habitat area and total suitable habitat area are increased due to the future climatic change scenario. highly suitable habitat of Satyrium's shows the shifting to the areas with high altitude. Satyrium's habitat loss of suitable areas showing the increase in the emission concentrations and range of increased area also showing the increase trend in population, making the area larger than the area lost.

\section{Competing Interests Statement}

The authors declare that they have no known competing interest or personal relationships that could have appeared to influence the work reported in the paper.

\section{Data Availability Statement}

All authors agreed to deposit data from this manuscript to a public repository.

Data are submitted to Dryad, and DOI number is https: // doi.org / 10.5061 /dryad. k98sf7m6v.

\section{References}

1. Bayer AD, Fuchs R, Mey R, Krause A, Verburg PH, Anthoni P, et al. Diverging land-use projections cause large variability in their impacts on ecosystems and related indicators for ecosystem services. Earth System Dynamics. 2021;12(1):327-51.

2. Allen SK, Plattner G-K, Nauels A, Xia Y, Stocker TF. Climate Change 2013: The Physical Science Basis. An overview of the Working Group 1 contribution to the Fifth Assessment Report of the Intergovernmental Panel on Climate Change (IPCC). May 01, 20142014. p. 3544.

3. Bellard C, Bertelsmeier C, Leadley P, Thuiller W, Courchamp F. Impacts of climate change on the future of biodiversity. Ecology letters. 2012;15(4):365-77.

4. Kumar D, Rawat S, Joshi R. Predicting the current and future suitable habitat distribution of the medicinal tree Oroxylum indicum (L.) Kurz in India. Journal of Applied Research on Medicinal and Aromatic Plants. 2021;23:100309.

5. Araujo MB, Peterson AT. Uses and misuses of bioclimatic envelope modeling. Ecology. 2012;93(7):152739.

6. Merow C, Smith MJ, Silander Jr JA. A practical guide to MaxEnt for modeling species' distributions: what it does, and why inputs and settings matter. Ecography. 2013;36(10):1058-69.

7. Pearson RG, Raxworthy CJ, Nakamura M, Townsend Peterson A. ORIGINAL ARTICLE: Predicting species distributions from small numbers of occurrence records: a test case using cryptic geckos in Madagascar. Journal of Biogeography. 2007;34(1):102-17.

8. Phillips SJ, Anderson RP, Schapire RE. Maximum entropy modeling of species geographic distributions. Ecological Modelling. 2006;190(3):231-59.

9. Mahendran G, Bai VN. Mass propagation of Satyrium nepalense D.Don.-A medicinal orchid via seed culture. Scientia Horticulturae. 2009;119(2):203-7. 
10. Cun Y. A Study on the Reproductive Ecology of Satyrium ciliatum Ldl. (Orchidaceae): Chinese Academy of Sciences; 2005.

11. Fick SE, Hijmans RJ. WorldClim 2: new 1-km spatial resolution climate surfaces for global land areas. International Journal of Climatology. 2017;37(12):4302-15.

12. Zhou Y, Zhang Z, Zhu B, Cheng X, Yang L, Gao M, et al. MaxEnt Modeling Based on CMIP6 Models to Project Potential Suitable Zones for Cunninghamia lanceolata in China. Forests. 2021;12(6).

13. Riahi K, van Vuuren DP, Kriegler E, Edmonds J, O'Neill BC, Fujimori S, et al. The Shared Socioeconomic Pathways and their energy, land use, and greenhouse gas emissions implications: An overview. Global Environmental Change. 2017;42:153-68.

14. Graham MH. CONFRONTING MULTICOLLINEARITY IN ECOLOGICAL MULTIPLE REGRESSION. Ecology. 2003;84(11):2809-15.

15. Pan J, Fan X, Luo S, Zhang Y, Yao S, Guo Q, et al. Predicting the Potential Distribution of Two Varieties of Litsea coreana (Leopard-Skin Camphor) in China under Climate Change. Forests. 2020;11(11).

16. Fielding AH, Bell JF. A review of methods for the assessment of prediction errors in conservation presence/absence models. Environmental Conservation. 1997;24(1):38-49.

17. Xu D, Zhuo Z, Wang R, Ye M, Pu B. Modeling the distribution of Zanthoxylum armatum in China with MaxEnt modeling. Global Ecology and Conservation. 2019;19:e00691.

18. Janitza S, Strobl C, Boulesteix A-L. An AUC-based permutation variable importance measure for random forests. BMC Bioinformatics. 2013;14(1):119.

19. Etherington TR. Python based GIS tools for landscape genetics: visualising genetic relatedness and measuring landscape connectivity. Methods in Ecology and Evolution. 2011;2(1):52-5.

20. Brown JL, Bennett JR, French CM. SDMtoolbox 2.0: the next generation Python-based GIS toolkit for landscape genetic, biogeographic and species distribution model analyses. PeerJ. 2017;5:e4095.

21. Mishra AP, Saklani S. Satyrium nepalense: a rare medicinal orchid of western Himalaya (India); phytochemical screening, antimicrobial evaluation and conservation studies. Indonesian Journal of Pharmacy. 2012;23(3):162-70.

22. Feng L, Sun J, Shi Y, Wang G, Wang T. Predicting Suitable Habitats of Camptotheca acuminata Considering Both Climatic and Soil Variables. Forests. 2020;11(8).

23. Li HQ, Liu XH, Wang JH, Xing LG, Fu YY. Maxent modelling for predicting climate change effects on the potential planting area of tuber mustard in China. The Journal of Agricultural Science. 2019;157(5):375-81.

24. Thomas CD, Cameron A, Green RE, Bakkenes M, Beaumont LJ, Collingham YC, et al. Extinction risk from climate change. Nature. 2004;427(6970):145-8.

25. Johnson SD, Peter CI, Ellis AG, Boberg E, Botes C, van der Niet T. Diverse pollination systems of the twin-spurred orchid genus Satyrium in African grasslands. Plant Systematics and Evolution. 2011;292(1):95103.

26. Deblauwe V, Droissart V, Bose R, Sonke B, Blach-Overgaard A, Svenning JC, et al. Remotely sensed temperature and precipitation data improve species distribution modelling in the tropics. Global Ecology and Biogeography. 2016;25(4):443-54.

27. Wang R, Jiang C, Liu L, Shen Z, Yang J, Wang Y, et al. Prediction of the potential distribution of the predatory mite Neoseiulus californicus McGregor in China using MaxEnt. Global Ecology and Conservation. 2021;29:e01733. 
28. Warren DL, Dornburg A, Zapfe K, Iglesias TL. The effects of climate change on Australia's only endemic Pokemon: Measuring bias in species distribution models. Methods in Ecology and Evolution. 2021;12(6):98595.

29. Ghini R, Hamada E, Angelotti F, Costa LB, Bettiol W. Research approaches, adaptation strategies, and knowledge gaps concerning the impacts of climate change on plant diseases. Tropical Plant Pathology. $2012 ; 37: 5-24$.

30. Wang JL, Wang HJ. Research on suitable habitat distribution for Chrysolophus pictus based on the MaxEnt model. Anhui Forestry Science and Technology. 2016;42(1/2):12-5.

31. Jia X, Ma F, Zhou W, Zhou L, Yu D, Qin J, et al. Impacts of climate change on the potential geographical distribution of broadleaved Korean pine (Pinus koraiensis) forests. Acta Ecologica Sinica. 2017;37(2):464-73.

32. Luo C, Shen Z, Li Y, Han J, Pausas JG, Xu Q, et al. Determinants of post-fire regeneration demography in a subtropical monsoon-climate forest in Southwest China. Science of the Total Environment. 2021;766:142605.

33. Fu G, Xu X, Ma J, Xu M, Liu J, Ding A. Responses of Haloxylon ammodendron potential geographical distribution to the hydrothermal conditions under MaxEnt model. Pratacultural Science. 2016;33:2173-9.

34. Hu X, Wu FC, Guo W, Liu N. Identification of potential cultivation region for Santalum album in China by the MaxEnt ecologic niche model. Scientia Silvae Sinicae. 2014;50(5):27-33.

\section{Hosted file}

Fig.doc available at https://authorea.com/users/444621/articles/544321-predicting-thepotential-distribution-of-endangered-and-rare-satyrium-species-in-china-under-climatechange 